\title{
Cyber-Harassment Victimization Among South African LGBTQIA+ Youth
}

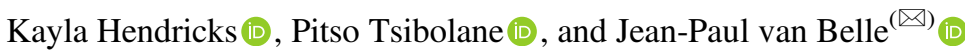 \\ University of Cape Town, Cape Town, South Africa \\ hndkay004@myuct.ac.za, \{pitso.tsibolane, \\ jean-paul.vanbelle\}@uct.ac.za
}

\begin{abstract}
Cyber-harassment victimization is one of today's major problems affecting the wellbeing of youth, particularly those that identify as lesbian, gay, bisexual, transgender, queer/questioning, intersex, asexual and gender nonconforming (LGBTQIA+). This exploratory study aims to determine the nature of cyber-harassment victimization, its enablers, and the coping mechanisms that online platforms provide to prevent or stop cyber-harassment. An online survey of ninety $(n=90)$ LGBTQIA+ young adults of ages between 18 and 34 from South Africa reveals a high incidence of exclusion, outing and harassment, covering a wide variety of types, duration and experienced severity, taking place through text messaging and social media sites such as Facebook, Instagram and Twitter. Most LGBTQIA+ youth resort to measures such as blocking, deleting offensive content and adjusting privacy settings to cope with cyber-victimization. Worryingly, the most severe effects of harassment such as depression, drug abuse, self-harm and suicide contemplation, have significant correlations with the harassment type used, harassment duration and harassment frequency. The paper discusses the implications for educational and social practice and future studies.
\end{abstract}

Keywords: Cyber-harassment $\cdot$ Cyber victimization $\cdot$ LGBTQIA+ $\cdot$ Negative impact of social media

\section{Introduction}

One of the negative impacts due to the rapid growth in social media access and connectivity is online harassment [21]. Studies show that LGBTQIA+ youth experience higher levels of cyber-harassment victimization than their non-LGBTQIA+ peers [14]. Despite intense social, cultural and political challenges, academic research into harassment and victimization amongst LGBTQIA+ individuals in Africa is growing [14] but gaps exist, particularly in the area of online harassment victimization. Research into the rate of victimization among LGBTQIA+ individuals in South Africa shows that prejudice based on sexual orientation ranks as the second highest form of discrimination, with prejudice based on nationality rank as the most prevalent form [14] but it is not clear what the levels and the nature of online based harassment victimization looks like.

This study, therefore, aims to address this research gap regarding the online harassment of LGBTQIA+ youth in South Africa. The study uses exploratory quantitative 
research to gain a better understanding of this problem. The overall goal of this study is to determine the nature of cyber-harassment victimization, its enablers, and the coping mechanisms that online platforms provide to prevent or stop cyber-harassment. The three questions explored are:

- What is the current nature and level of cyber-harassment victimization among LGBTQIA+ youth in South Africa?

- Which aspects of online platforms enable cyber-harassment victimization of South African LGBTQIA+ youth?

- Which aspects of online platforms afford LGBTQIA+ youths coping mechanisms against cyber-harassment victimization?

\section{Literature Review}

Online social networking has seen a vast period of growth in the past two decades globally [9, 13, 19]. The wide range of communication channels including emails, instant messengers, text messages, social networking sites, blogs, wikis and chat rooms continues to fuel this growth [9, 13]. The use of social networking technologies is a convenient way for the youth to explore their identity, better social skills and to improve media literacy [8]. Despite the many benefits attributed to the rapid growth of social networking technologies, it has also been associated with serious undesirable social implications, such as cyber-harassment victimization [21].

\subsection{Cyber-Harassment}

Cyber harassment affects individuals of different age groups and is a prevalent cause for concern linked to negative social effects such as depression and suicide [16, 21]. While cyber-harassment is an extension of traditional harassment, various definitions of cyber-harassment exist. There are two forms of cyber-harassment, direct/physical and direct [16]. Direct cyber harassment consists of physical methods such as the sending of viruses, threatening verbal messages and nonverbal methods, which could include the sending of offensive or explicit images, as well as social methods, which include censoring or kicking an individual out of an online group. Indirect cyber-harassment comprises of online gossip around the subject of the individual and taking part in activities such as commenting or voting on insulting websites.

More formally, cyber harassment refers to "any behavior performed through electronic or digital media by individuals or groups that repeatedly communicates hostile or aggressive messages intended to inflict harm or discomfort on others" [21]. Victims of cyber harassment are often victims of traditional harassment [20]. However, cyber harassment has emerged as a significant issue because of its rapidly evolving digital nature. Cyber-harassment differs from traditional harassment in many respects such its potential anonymity, being unconstrained by time, larger audience size, lack of physical interaction, high frequency of violation, the variety of media that can be used and the reduced threat of intervention [16]. Some of the common forms of cyberharassment include the use of swear words, various insults, unwelcome jokes, fake 
names, teasing, spreading rumors, humiliating and making physical threats, with female students specifically using methods such as gossiping and using attacks that are personal to the individual [19]. A categorization of cyber-harassment outlines eight different types of victimization [22].

Table 1. Types of cyber-harassment victimization

\begin{tabular}{l|l}
\hline $\begin{array}{l}\text { Cyber- } \\
\text { harassment }\end{array}$ & Description \\
\hline Flaming & Engaging online arguments usually involving unfounded personal attacks \\
\hline Impersonation & Pretending to be another in order to inflict harm \\
\hline Denigration & The spreading of offensive information about a person \\
\hline Exclusion & $\begin{array}{l}\text { Deliberately removing or leaving out an individual in an online group } \\
\text { setting }\end{array}$ \\
\hline Outing & Sharing of an individual's confidential information with outside parties \\
\hline Trickery & Deceiving an individual into sharing confidential information \\
\hline Cyberstalking & Threating or harassing an individual \\
\hline Sexting & Sending sexually inappropriate and offensive images to an individual \\
\hline
\end{tabular}

New descriptors that form part of the cyber-harassment victimization types are "trolling" and "griefing" [19]. Trolling is the act of making random unsolicited and/or controversial comments on various online social networking internet forums with the intent to provoke an emotional knee jerk reaction from unsuspecting readers to engage in a fight or argument. Griefing is performing actions in an online game for instance, to prevent another individual from enjoying the game i.e. causing them 'grief' [19].

Factors Linked to Victimization. Victims, in the context of this study are those who report they are the target of cyberbullying. This study makes a link between these victims and several characteristics that are common amongst them and could have an impact on the likelihood of victimization. Studies have shown factors such as comparative physical weakness, fear of aggressive behavior, more trusting and open behavior, and poor social skills and low popularity [5]. In addition, in comparison to traditional harassment, the cyber-harassment victimization rates are higher for females than they are for males $[9,13]$. However, in contrast to these findings, other studies show that demographic factors such as age and gender do not seem to provide a clear link to victimization prevalence [21]. Shyness is a potential contributory factor to cybervictimization but there is still clear evidence to isolate shyness as a victimization characteristic specifically as it could be the consequence of cyber harassment [1]. Similarly, forming relationships with strangers is a factor that is more prevalent amongst victimized youth [13]. Following on from that notion, much of the studies developed around the topic of cyber-harassment victimization, as well as their instruments of measurement, consist of inadequate, empirically limited findings, which further extensive research can illuminate [14]. 
Enabling Factors. Anonymity or the ability to hide or falsify an individual's real identity, a capability that comes with various online platforms, enables cyber-harassment. Most cyber-harassment victimization is largely anonymous, and this factor enables hostile and thoughtless behavior intended to instill fear and feelings of distress into victims [2]. The lack of physical interaction may lead to individuals acting in ways that they would not if they were in the public eye, and this relates to the extent to which an individual is at ease behind the relative safety of their communicative technologies [13]. The larger audience that is accessible through the click of a button in comparison to traditional harassment methods also enables cyber-harassment victimization.

The lack of interference by authority figures such as parents and teachers is often much more pronounced in cyber harassment victimization incidents than in comparison to traditional harassment [2]. The notion of free speech also increases the likelihood of individuals feeling like they are able to communicate any content that they feel is necessary online, which has been associated with increases in online harassment [9].

Impact on Victims. There have been numerous negative impacts linked to the aftermath of cyber harassment victimization namely social, psychological, emotional and academic [21]. These effects could differ in severity ranging from "trivial levels of distress and frustration" to more serious mental or life problems such as deteriorating grades to difficulties concerning home life [21]. Absence from school is more prevalent amongst youth who are cyber-victimized as well as depression is also common among youth who are victims of cyber-harassment [2, 5]. Anxiety, low empathy, declining confidence levels, rejection by peers, substance abuse and aggression are additional factors positively associated with victimization effects [5, 16]. Cyber-harassment worsens the intensity pre-existing negative emotions such as hopelessness and low selfesteem particularly among young people [5, 16]. Other studies show a rise of the incidence in self-harm and suicidal ideation due to cyber-victimization among youth that struggle with hopelessness and self-esteem [19].

Mitigation Plans and Safeguards. Research show several technological coping mechanisms that serve as means to mitigate the negative effects of concerning cyberharassment [21]. The nature or type of these mechanisms differs from case to case depending on the severity of harassment experienced by a victim [21]. These coping mechanisms include blocking and deletion of offender/offensive messages; adjusting to more strict privacy settings; removing offensive content; changing of username; changing of email address; avoidance of technology; changing of number; changing of passwords; tracking of IP addresses; contacting of site administrators; responding to the offender online and bystanders defending victims [2, 16, 19].

\subsection{Context: The LGBTQIA+ Community in South Africa}

The LGBTQIA+ community is a collective term referring to lesbian, gay, bisexual, transgender, queer, questioning, asexual, and other identities that are not heterosexual and/or cisgender [11]. Research concerning the LGBTQIA+ community in Africa is still developing. This is not due to the lack of prevalence but rather attributable to the 
lack of social and legislative acceptance that surrounds the topic [7]. This is borne by the fact that several African countries have some law criminalizing either homosexuality or an aspect of it.

While several African countries do not recognize the LGBTQIA+ community, South Africa seems to be the relative exception [17]. South Africa's post-apartheid constitution prohibits discrimination on the grounds of sexual orientation and legalizes same sex marriage [4]. However, while the state is fully accepting of non-heterosexual sexuality, the attitude of some members of the population are still discriminatory of homosexuality. Surveys show that the populace still highly values heteronormativity and remains deeply conservative, only marginally accepting of homosexuality. Many South Africans still harbor a judgmental outlook towards the LGBTQIA+ community [4]. The negative attitudes and discrimination surrounding the LGBTQIA+ community were central to the study conducted by the Hate Crimes Working Group [15]. This study also observed that most of the community rhetoric, harassment or hate crime incidents take place through social media platforms or electronic communication [14, 15]. There is therefore a need for a greater number of studies investigating different aspects of online discrimination concerning LGBTQIA+ individuals.

\subsection{Theoretical Framework}

The theoretical framework chosen for this study combined aspects of two behavioral theories: The Lifestyle Exposure Theory and The Social Presence Theory. The theoretical constructs enabled insights into aspects of victimization, behavior and the detrimental factors of cyber harassment concerning individuals in the LGBTQIA+ community.

The Lifestyle Exposure Theory aims to understand if specific lifestyles are associable with different probabilities of victimization [3]. It suggests that due to certain demographic profiles, certain people are more at risk of victimization due to the perceived lifestyles risks [12]. It posits a link between both the lifestyle and demographics of individuals and the types of victimization potential. The Lifestyle Exposure Theory lends itself particularly to why the LGBTQIA+ community is at risk for cyber harassment victimization. The role played by the peer pressure in the victimization of LGBTQIA+ is also considered [10].

The Social Presence Theory posits that the extent to which a person perceives another as a real person (presence) in mediated communication (such as online communication) varies according to the quality of the medium used [6]. This quality of the medium includes the extent to which the medium conveys information about facial expression, direction of looking, posture, dress and nonverbal cues [6]. The Social Presence Theory helps in the identification of factors that could be more detrimental in cyber harassment rather than traditional face-to-face harassment.

\section{Research Methodology}

The study aims to determine the nature of cyber-harassment victimization, its enablers, and the coping mechanisms that online platforms provide to prevent or stop cyberharassment. The study is both descriptive and exploratory [18]. The research used a 
quantitative survey approach to obtain the empirical data. The target population of this study are young individuals (18 to 35 years) in the LGBTQIA+ community. The survey instrument was developed based on the pre-validated questions from the Lifestyle Exposure Theory study [12] as well The Social Presence Theory study [6]. Face validity was conducted with 3 experts who are also gender activists on the resulting survey. We distributed a mass email, requesting participants for the study to the members of a large academic university in South Africa. Requests to participate in the study were distributed through social media sites such as Facebook and Twitter to the public. The study employed the three theories to enable the testing of certain statements contained in the literature as well as to develop the survey instrument. The University's ethics committee approved the research and the survey instrument. Respondents are anonymous and were able to opt out at any time during the online survey. A number of direct 24/7 help line numbers, email addresses and details of the University's student help as well as relevant NGOs dedicated to the LGBTQIA+ community were listed in case respondents wanted psychological or any other assistance during or after the survey.

\section{Research Findings and Discussion}

\subsection{Demographics}

The sample consisted of 90 valid responses of which $2 \%(n=2)$ of respondents identified as asexual, 29\% $(\mathrm{n}=26)$ as bisexual, $36 \%$ as homosexual (gay or lesbian), $19 \%(\mathrm{n}=17)$ as pansexual, $2 \%(\mathrm{n}=2)$ as plus $(+)$ and $12 \%(\mathrm{n}=11)$ as queer. The age distribution was positively skewed with the largest response from the 18-25 years age group representing $94 \%(n=85)$ of the respondents and only $2 \%(n=2)$ between the ages of 25 and 30 and $3 \%(n=3)$ being between the age of 30 and 35 .

Of the valid responses, $82 \%(\mathrm{n}=74)$ of LGBTQIA+ individuals reported to have been harassed due to their orientation while $18 \%(n=16)$ reported to have never been harassed online due to their sexual orientation. We note that there may be a response bias i.e. those that were harassed may have a higher inclination to complete the survey.

\subsection{Research Question 1: "What Is the Current Nature and Level of Cyber-Harassment?"}

The types of harassment experienced by members of the LGBTQIA+ community are depicted in Fig. 1 (left). Being outed is the highest ranked type of cyber-harassment experienced by most respondents, followed by harassment, exclusion, flaming and denigration. Figure 1 (right) shows the online mediums on which LGBTQIA+ individuals most commonly experience cyber-harassment. Text-based harassment seems to be the most prevalent, followed by Facebook, Instagram and Twitter. Text-based services include SMS (Short Message Service) and WhatsApp are the most affordable and commonly used services in South Africa. 

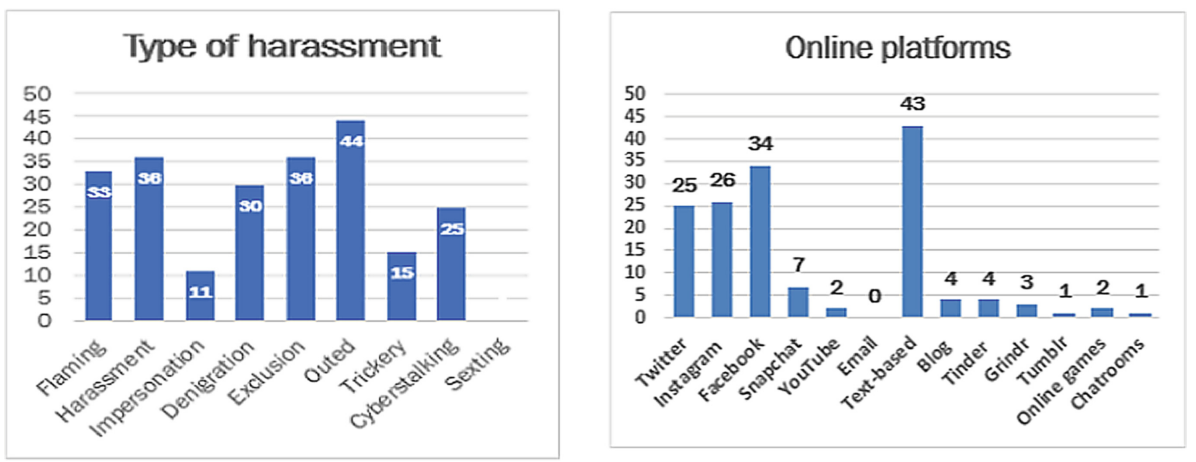

Fig. 1. The occurrence of types of cyber-harassment and the associated online platforms

\subsection{Research Question 2: "Which Aspects of Online Platforms Enable Cyber-Harassment?"}

The severity of the harassment experienced by individuals in the LGBTQIA+ community in Fig. 2 follows a normal distribution, with the majority of respondents experiencing a medium severity level (3) and fewer respondents on the outer more extreme ends of the rankings (the ranking system ranges from 1 being 'not severe at all' to 5 being 'very severe').

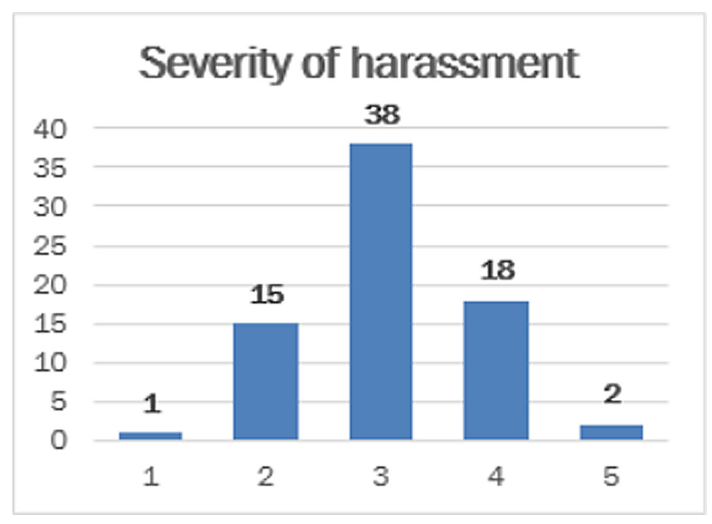

Fig. 2. Severity of harassment

The duration of harassment in the research instrument ranged from 1 being brief to 5 being years. The results indicate that there is an even spread amongst duration ranks, with the "brief" ranking being most prevalent amongst respondents (Fig. 3). 


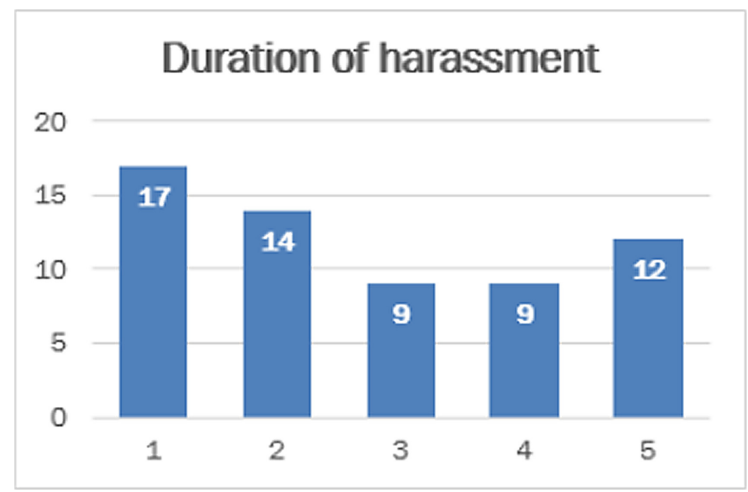

Fig. 3. Duration of harassment

The majority of the cyber-victimization incidents $(66 \%)$ that members of the LGBTQIA+ community experienced were perpetrated by people that were identifiable i.e. not anonymous. Only $9 \%$ of the perpetrators were anonymous and $25 \%$ were sometimes anonymous. This contradicts research that anonymity is as an enabler of cyber harassment $[2,13]$. Most cyber-harassment incidents involved an online audience (44 out of 72). This seems to support the finding that ease of spread is an enabling factor for cyber harassment [16].

Most incidents, 57 out of $74(77 \%)$, were not reported with the authorities for further action. The fact that victims do not seem to report incidents could potentially lead to the reason why offenders show a lack of fear of being caught. This could contribute to the high rates of harassment in the LGBTQIA+ community [2, 13]. Most incidents were experienced in the evening (55\%), with afternoon the second-most (34\%). Only $3 \%$ of the incidents were in the morning, $9 \%$ occurring at any time of the day. This result aligns with prior research findings that the incidence of cyberharassment increases in the evenings $[16,21]$.

\subsection{Research Question 3: "Which Aspects of Online Platforms Are Considered as Coping Mechanisms Against Harassment?"}

Figure 4 depicts the types of safeguards used by members of the LGBTQIA+ community to prevent/stop cyber-harassment. Blocking (where the victim stops the abuser from accessing their profile) ranks as the most prevalent type of safeguard utilized by cybervictims. The deletion of the offensive content (the victim removing abusive messages) follows. The adjustments of settings (e.g. making the victim's account private or inaccessible by the abusers) follows closely. Other coping mechanisms such as responding back, deleting own content and withdrawal from technology are also common. Our research does not give conclusive evidence on how effective the following coping mechanisms/safeguards are in the prevention or remedy of cyber harassment [21]. 


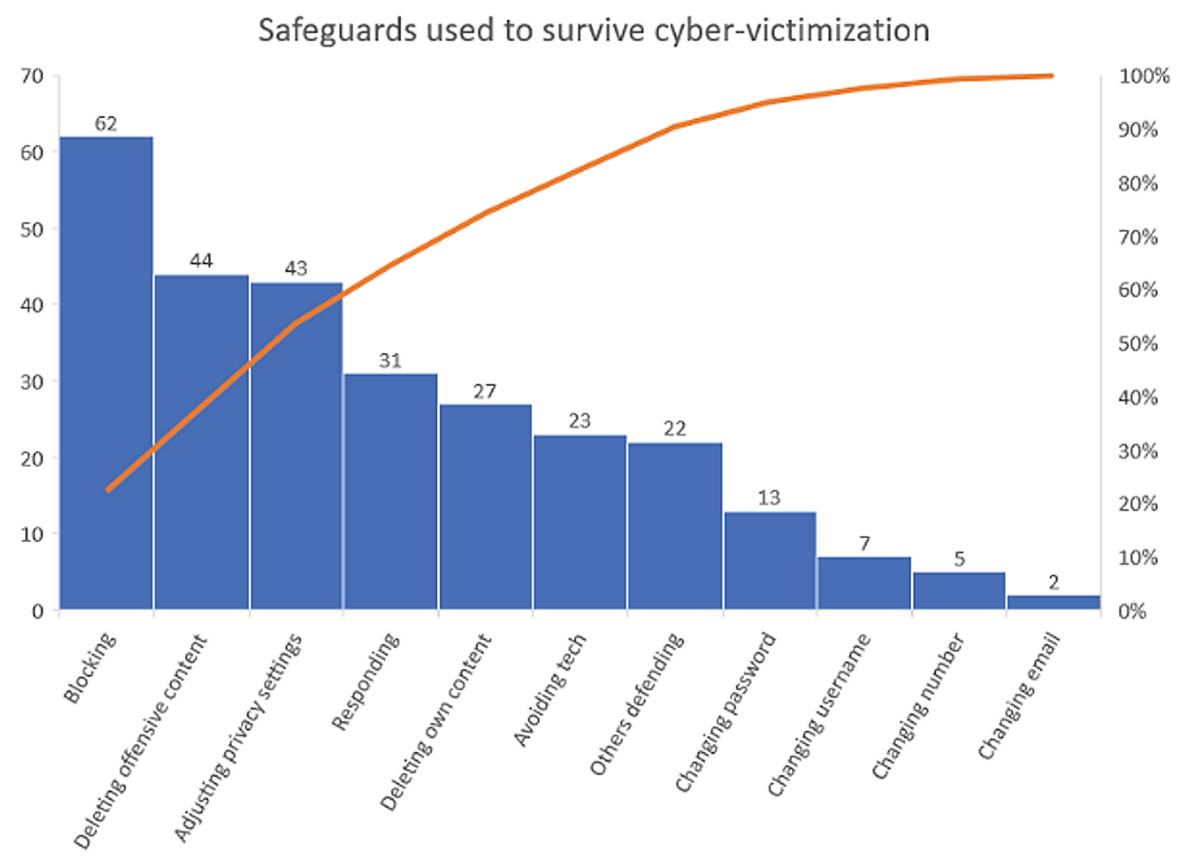

Fig. 4. Safeguards used to prevent/stop harassment

\subsection{Inferential Statistics: Harassment Effects by Harassment Type and Attribute}

To test the differences in harassment effects based on harassment type, each harassment effect and harassment type were grouped into two categories (Yes, No) and tested against one another. We used a Chi-Squared test to examine any relationships. The pvalue associated with each Chi-Square statistics is listed in Table 1, with the significant values $(\mathrm{p}<0.05)$ highlighted. For the harassment attributes duration, severity and frequency (bottom three rows in Table 2) the Pearson correlation coefficient was used as the relevant test statistic.

The test revealed a number of significant associations, although some of these may be an artefact of the data. However, the harassment types (reading by row) of denigration, exclusion and cyber-stalking seem to have the biggest impacts, as do the harassment duration, as well as frequency. Extremely worrying is the fact that the most significant impacts (reading by column) seem to be quite severe: depression, substance abuse, selfharm and suicide contemplation all have at least three significant correlations. 
Table 2. Harassment type/attributed and their impact (p-value based on test-statistic)

\begin{tabular}{|c|c|c|c|c|c|c|c|c|c|c|c|}
\hline $\begin{array}{l}\text { Effect } \\
\text { experienced as a } \\
\text { consequence } \\
\text { from: } \\
\text { Harassment type }\end{array}$ & 䓌 & 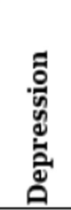 & 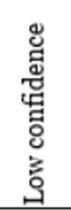 & 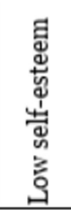 & 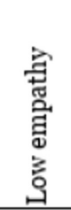 & 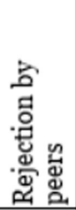 & 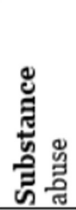 & 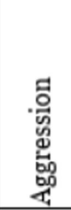 & 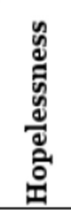 & 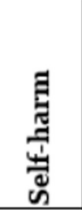 & 突 \\
\hline Flaming & .897 & $.73 \circ$ & .363 & .776 & .473 & .211 & .624 & .175 & .251 & .430 & .850 \\
\hline Harassment & .271 & .253 & .247 & .345 & .146 & .506 & .843 & .306 & .511 & .339 & .151 \\
\hline Impersonation & .088 & .618 & .377 & .601 & .333 & .331 & .423 & .076 & .763 & .713 & .524 \\
\hline Denigration & .089 & .004 & .848 & .299 & .359 & .171 & .020 & .650 & .891 & .004 & .018 \\
\hline Exclusion & .271 & .107 & .011 & .159 & .599 & .000 & .025 & .843 & .032 & .039 & .000 \\
\hline Outed & .391 & .056 & .219 & $.18_{3}$ & .980 & .938 & .429 & .650 & .891 & .028 & .787 \\
\hline Trickery & .112 & .406 & .132 & .204 & .975 & $.53^{8}$ & .611 & .611 & .202 & .840 & .281 \\
\hline Cyber-stalking & .683 & .555 & .936 & .369 & .199 & .665 & .092 & .092 & .041 & & .001 \\
\hline \multicolumn{12}{|c|}{ Harassment attribute } \\
\hline Duration & .387 & .180 & .220 & .025 & .637 & .024 & .043 & .707 & .465 & .121 & .068 \\
\hline Severity & .215 & .039 & .239 & .14 .5 & .676 & .305 & .957 & .361 & .058 & .105 & .290 \\
\hline Frequency & .073 & .044 & .636 & .241 & .290 & .466 & .496 & .090 & .118 & .028 & .117 \\
\hline
\end{tabular}

\section{Conclusion}

Cyber harassment is a growing topic of research due to the rapid rate of the technology advancement society is currently experiencing. However, research concerning minority groups such as LGBTQIA+ and the cyber harassment that those groups incur is severely lacking. The overall aim of this study is to highlight that cyber harassment in minority groups is a topic of importance worthy of more academic research.

The study's results show that majority of the LGBTQIA+ group have been cyberharassed. The most prevalent type of cyber harassment seems to be the outing of individual, and the platform most preferred by offenders seems to be text-based (SMS WhatsApp etc.). The cyber harassment seems to vary in frequency, duration and severity, with some significant correlations to certain effects experienced, such as depression.

The findings regarding accessibility, ease of spread and lack of fear of offender penalization align with previous literature in that those factors are enablers of cyberharassment. The study also identified the various coping mechanisms that LGBTQIA+ individuals tend to adopt to deal with cyber-victimization. Further research should be conducted to find rates of effectiveness on remedy and prevention.

The most worrying finding of this research was the severity of the effect of harassment. We found to be significant effects from some types of harassment (denigration, exclusion and cyber-stalking), and harassment duration, as well as frequency. These resulted in statistically significant levels of depression, substance abuse, selfharm and suicide contemplation. This highlights the importance of this research and motivates strongly for further research to in this space as well as the importance of regulating or monitoring social platforms. 


\section{Limitations and Further Research}

The major limitation with this study is response bias (respondents who have been harassed are more likely to respond to the survey) and the sampling approach. The majority of the participants were mostly university students as this sample was the most easily accessible. This may mean that the results may not be as general to the wider public as students represent a distinct age and educational group, and are perhaps more homogenous than the rest of the LGBTQIA+ population.

Further research needs to focus on further validation of this study's findings, how effective coping strategies provided by online platforms are in the remedy and prevention of cyber harassment, and harassment experiences in less liberal environments than South Africa. A longitudinal study with a control group could also yield critical insights.

\section{References}

1. Anon: Internet: Statistics and Market Data about the Internet (2017). https://www.statista. com/markets/424/internet/

2. Aricak, T., et al.: Cyberbullying among Turkish adolescents. Cyber-Psychol. Behav. 11(3), 253-261 (2008)

3. Dietrich, H.: Victimology: an emphasis on the lifestyle-exposure theory and the victim precipitation theory as it applies to violent crime, s.l.: s.n (2008)

4. Devji, Z.Z.: Forging paths for the African queer: is there an "African" mechanism for realizing LGBTIQ rights? J. Afr. Law 60(3), 343-363 (2016)

5. GottheilN, F., Dubow, E.F.: Tripartite beliefs models of bully and victim behavior. Bully. Behav.: Curr. Issues Res. Intervent. 2, 25-47 (2001)

6. Gunawardena, C.N.: Social presence theory and implications for interaction and collaborative learning in computer conferences. Int. J. Educ. Telecommun. 1(2/3), 147-166 (1995)

7. Inglehart, R., Welzel, C.: Modernization, Cultural Change, and Democracy: The Human Development Sequence. Cambridge University Press, Cambridge and New York (2005)

8. Jackson, C.: Your students love social media. Teach. Tolerance Issue 39, 38-41 (2011)

9. Kuzma, J.: Empirical study of cyber harassment amount social networks. Int. J. Technol. Hum. Interact. 9(2), 53-65 (2013)

10. LaMorte, W.W.: Behavioral change models: social norms theory. Boston University School of Public Health, s.l. (2016)

11. Locke, A., Lawthom, R., Lyons, A.: Social media platforms as complex and contradictory spaces for feminisms: visibility, opportunity, power, resistance and activism. Feminism Psychol. 29(1), 3-10 (2018)

12. Madero, A.: Lifestyle Exposure Theory of Victimisation. Wiley-Blackwell, Hoboken (2018)

13. Mishna, F., Khoury-Kassabri, M., Gadalla, T., Daciuk, J.: Risk factors for involvement in cyber bullying: victims, bullies and bully-victims. Child. Youth Serv. Rev. 32, 63-70 (2012)

14. Mitchell, Y., Nel, J.A.: The hate and bias crimes monitoring form project. The Hate Crimes Working Group, Johannesburg (2018)

15. OUT: New LGBTI hate crime reporting platform launched (2018). http://www.out.org.za/ index.php/what-s-hot/news/558-new-lgbti-hate-crime-reporting-platform-launched

16. Sabella, R.A., Patchin, J.W., Hinduja, S.: Cyberbullying myths and realities. Comput. Hum. Behav. 29, 1703-2711 (2013) 
17. Sandfort, T.G., Reddy, V.: African same-sex sexualities and gender-diversity: an introduction. Cult. Health Sex. 15(1), 1-6 (2013)

18. Saunders, M., Lewis, P., Thornhill, A.: The purpose of your research. In: Research Methods for Business Students, 5 edn., pp. 139-140. Pearson Education, Harlow (2009)

19. Slonje, R., Smith, P.K., Frisen, A.: Processes of cyberbullying, and feelings of remorse by bullies: a pilot study. Eur. J. Dev. Psychol. 9, 244-259 (2012)

20. Swearer, S.M., Hymel, S.: Understanding the psychology of bullying: moving toward a social-ecological diathesis-stress model. Am. Psychol. 70(4), 344-353 (2015)

21. Tokunaga, R.S.: Following you home from school: a critical review and synthesis of research on cyberbullying victimization. Comput. Hum. Behav. 26(3), 277-287 (2010)

22. Willard, N.E.: Cyberbullying and Cyberthreats: Responding to the Challenge of Online Social Aggression, Threats, and Distress. Research Press (2007) 